\title{
BMJ Open Patient work from a context and time use perspective: a mixed-methods study protocol
}

\author{
Kathleen Yin, ${ }^{1}$ Teresa Harms, ${ }^{2,3}$ Kenneth Ho, ${ }^{4}$ Frances Rapport, ${ }^{1}$ \\ Sanjyot Vagholkar, ${ }^{4}$ Liliana Laranjo, ${ }^{1}$ Enrico Coiera, ${ }^{1}$ Jonathan Gershuny, ${ }^{2}$ \\ Annie Y S Lau ${ }^{1}$
}

To cite: Yin K, Harms T, Ho $\mathrm{K}$, et al. Patient work from a context and time use perspective: a mixed-methods study protocol. BMJ Open 2018;8:e022163. doi:10.1136/ bmjopen-2018-022163

- Prepublication history and additional material for this paper are available online. To view these files, please visit the journal online ( $h$ ttp://dx.doi. org/10.1136/bmjopen-2018022163).

Received 4 February 2018 Revised 25 0ctober 2018 Accepted 1 November 2018

Check for updates

(c) Author(s) (or their employer(s)) 2018. Re-use permitted under CC BY-NC. No commercial re-use. See rights and permissions. Published by BMJ.

${ }^{1}$ Centre for Health Informatics, Australian Institute of Health Innovation, Macquarie University, Sydney, New South Wales, Australia

${ }^{2}$ Department of Sociology, Centre for Time Use Research, University of Oxford, Oxford, UK ${ }^{3}$ Planning and Transport Research Centre, Business School, University of Western Australia, Perth, Western

Australia, Australia

${ }^{4}$ Faculty of Medical and Health Sciences, Macquarie University, Sydney, New South Wales, Australia

Correspondence to

Dr Kathleen Yin;

kathleen.yin@mq.edu.au

\section{ABSTRACT}

Introduction Self-management is widely promoted but less attention is focused on the work required from patients. To date, many individuals struggle to practise self-management. 'Patient work', a concept that examines the 'work' involved in self-management, is an approach to understanding the tasks, effort, time and context from patient perspective. The purpose of our study is to use a novel approach combining non-obstructive observations via digital devices with in-depth qualitative data about health behaviours and motivations, to capture the full range of patient work experienced by people with type 2 diabetes and chronic comorbidities. It aims to yield comprehensive insights about 'what works' in selfmanagement, potentially extending to populations with other chronic health conditions.

Methods and analysis This mixed-methods observational study involves a (1) prestudy interview and questionnaires, (2) a 24-hour period during which participants wear a camera and complete a time-use diary, and a (3) poststudy interview and study feedback. Adult participants living with type 2 diabetes with at least one chronic comorbidity will be recruited using purposive sampling to obtain a balanced gender ratio and of participants using insulin and those using only oral medication. Interviews will be analysed using thematic analysis. Data captured by digital devices, diaries and questionnaires will be used to analyse the duration, time, context and patterns of health-related behaviours.

Ethics and dissemination The study was approved by the Macquarie University Human Research Ethics Committee for Medical Sciences (reference number 5201700718). Participants will carry a wallet-sized card that explains the purpose of the study to third parties, and can remove the camera at any stage. Before the poststudy interview begins, participants will view the camera images in private and can delete any images. Should any images be used in future publications or presentations, identifying features such as human faces and names will be obscured.

\section{INTRODUCTION}

Self-management is widely promoted but less attention is focused on the work required from patients. It is a constant commitment of learning new skills, breaking old habits

\section{Strengths and limitations of this study}

- This is the first study that employs a mixed-methods design using passive digital observation equipment, self-report time-use diaries, complemented with interviews and questionnaires, to document patient work.

- Using the body-worn camera may diminish the observer effect caused by having researchers observe participants in a private setting, as well as reducing the participant burden of having researchers in their homes for prolonged periods of time.

- The self-report diary complements data from the passive device by creating a holistic view of patient work carried out by the participant.

- The study is limited by the 24-hour data collection period, which may not include patient work that occurs frequently, but outside the observation period.

and managing different emotions over the length of the illness. ${ }^{1}$ Patients and their families often need to adjust their daily routines to accommodate for their health condition, such as making time to administer medications, visit medical professionals or prepare special meals. ${ }^{2-5}$ Yet, many individuals struggle to practise self-management effectively, especially for those with long-term chronic conditions. ${ }^{6-8}$

Past studies reported on the barriers and challenges to self-management, focusing on patients with multiple chronic conditions, ${ }^{67}$ the difficulty involved in self-management, ${ }^{8}$ time invested, ${ }^{9}$ competing priorities, ${ }^{10}{ }^{11}$ support sources required ${ }^{12}$ and how self-management differs between the patients' and healthcare professionals' perspectives. ${ }^{13}$ Yet, few studies reported on the actual 'work', or the tasks involved, from the patient, as well as whether self-management fits within the patient's context.

When self-management is poorly integrated into people's lives, the work required has the potential to generate additional stress, 
contributing to non-compliance and negatively impacting on patient health outcomes. ${ }^{14}{ }^{15}$ Unfortunately, many health interventions are currently implemented without considering the work involved from patient perspective or the context of a patient's life. Treatment and self-management recommendations, while given with goodwill, often fail to acknowledge the specificities of a patient's daily routine, physical environment, social obligations, as well as internal beliefs and attitudes. ${ }^{16-19}$ In parallel, digital health interventions hold the promise to improve self-management. However, numerous studies evaluating digital self-management interventions showed a high participant dropout rate, with some studies having retention rates of $1 \%$ by the end of the study period. ${ }^{16}{ }^{19-21}$ Specifically, participants reported the devices' lack of 'fit' into their daily lives as reason for discontinuation. ${ }^{22}$

Understanding whether there is a dissonance between patients' existing routines and the work involved in self-management is important to overcome barriers and challenges. The concept of 'patient work', derived from health ergonomics, describes the tasks (physical and cognitive) conducted by patients to manage their health, as well as the holistic sum of contexts (physical, social, mental and organisational) that influence the work conducted ${ }^{23-25}$ It is an approach to understanding the actions people take (or not take) in self-management. While self-management focuses on the strategies people employ, patient work breaks down these strategies into day-to-day tasks and examines how the effort and time involved, as well as the contextual and ergonomic factors, affect the way self-management is practised and why some tasks are carried out while others are neglected.

The purpose of this study is to examine the patient work involved in living with multiple chronic conditions, focusing on the daily lives of people with type 2 diabetes and chronic comorbidities.

\section{Patient work research}

Previous patient work research had focused on patients living with cardiovascular diseases such as heart failure and stroke. ${ }^{24-26}$ However, no studies have focused specifically on people living with more than one chronic condition.

In addition, previous patient work approaches relied heavily on self-report methods (interviews ${ }^{27}{ }^{28}$ ), direct observation (researchers being present continuously in participants' homes) or theoretical modelling (analysing macroergonomic elements). ${ }^{29} 30$ These data collection methods are limited by the level of reliability in self-report data (eg, social desirability effect) and the degree of discomfort participants experience during direct observation.

The need for direct observation by researchers also meant the duration of visits had to be limited, ranging from $30 \mathrm{~min}$ to 1 hour at a time, ${ }^{30}$ making these short periods unlikely to capture the 'natural' routines of participants as there is high likelihood they could be affected by the observer effect. ${ }^{31}$ To our knowledge, no studies have used digital devices that automatically and non-obstructively collect data on patient work over a longer time frame.

\section{Type 2 diabetes and other comorbidities}

The nature of type 2 diabetes, which impacts the entire metabolic process, means that patients need to carry out a variety of different activities. These may include diet modification, ${ }^{32}$ weight management, ${ }^{33}$ exercise, ${ }^{34}$ complication prevention, ${ }^{35}$ blood glucose tests ${ }^{36}$ and insulin injections. ${ }^{26}$

Metabolically related comorbidities such as hyperlipidaemia, cardiovascular diseases and renal disorders $^{3738}$ are frequently present in people living with type 2 diabetes, warranting the inclusion of comorbidities to reflect the true extent of patient work. Comorbidity is also frequently a criterion for exclusion in research studies, suggesting this study could give rare insights into the lives of people living with type 2 diabetes, as well as a greater understanding of self-management in patients with chronic comorbid conditions.

\section{AIMS}

This study will investigate the patient work conducted by people living with type 2 diabetes and at least one chronic comorbidity. It will address three gaps identified in the literature: the few studies reporting the 'work' involved in self-management from patient perspective (patient work), the methodological gap in using digital devices to collect data non-obstructively on self-management activities and contexts over a longer time frame ( 24 hours period) and focusing on people with complex challenges to self-management (those with multiple chronic conditions).

\section{METHODS}

This is a mixed-methods observational study that involves three stages: (1) prestudy interview and questionnaires, (2) a 24-hour period during which participants wear a camera and complete a time-use diary, and a (3) poststudy session with interview and study feedback. Our study adapted the protocols used and validated by Kelly et $a l,{ }^{39} 40$ which were employed in research projects conducted by the Centre for Time Use Research and the British Heart Foundation Centre on Population Approaches for Non-Communicable Disease Prevention, both at the University of Oxford.

\section{Sample and recruitment}

Eligible participants are: (1) aged 18 years and older; (2) consenting adults who can speak, write and read in English; (3) have been diagnosed with type 2 diabetes and at least one chronic comorbidity; (4) willing to complete a time-use diary for 1 day; and (5) agree to wear the body-worn camera for 1 day. Exclusion criteria include those who are currently experiencing: (1) pregnancy; (2) critical illness; (3) end-stage disease; (4) 
dementia; (5) severe mental disorders that prevent one from giving informed consent; and (6) dependent on others for basic activities of daily living such as dressing and eating. Participants will receive $\$$ A60 of shopping vouchers as remuneration.

To capture patients at various stages of diabetes, 40 participants will be recruited, with balanced gender ratio, and balanced numbers of participants using only oral medication and those using insulin. The number of participants chosen was informed by previous qualitative studies in patient work. ${ }^{27} 28$ This sample size offers the power to achieve rich and nuanced data, while acknowledging the different time intervals between data points for each data capture tool. ${ }^{24} 2728$ Previous patient work studies analysed 60-80 hours of interviews or direct observations per study. ${ }^{27} 28$ This study will capture approximately 2 hours of interview data and approximately 5000 images per participant. Therefore, a sample size of 40 participants is comparable to the depth of data captured by previous studies.

Recruitment will be carried out via Macquarie University general practice and general practitioners (GP) and endocrinologists in the surrounding suburbs. These medical practitioners manage a large and diverse patient base across northern Sydney and cover a wide range of culturally and economically diverse population groups.

Promotional flyers will be distributed to the participating medical practices and includes general study information and a link to the study website, where potential participants can check the eligibility criteria (www.tinyurl. com/patientswork). The study will also be promoted on the Macquarie University Facebook page and via an emailed newsletter associated with the University.
Healthcare practitioners will not be notified if their patients agreed to participate in the study to reduce the possibility of coercion.

Databases of medical practices involved in recruitment will be searched to identify eligible patients for the study. Only personnel employed by the medical practices will conduct these searches. Participating healthcare practitioners will also send invitation letters to eligible patients, with a prepaid reply slip included. Patients interested in participating will be asked to return the reply slip with their contact details, so a member of the research team can contact them.

Telephone calls will also be made to eligible patients to inform them about the study. If during the initial phone call the potential participant agrees to consider participating, the researcher will send a detailed email to the participant with a digital version of the information pamphlet as well as a link to the study website. The potential participant then either agrees to participate or declines. If the potential participant agrees to participate, the researcher will arrange a suitable time for interviews to occur. All participants will provide written informed consent before data collection commences.

Initial contact for all participants will be a telephone call conducted by the research team, to check whether the participant fits the eligibility criteria, assure confidentiality and anonymity during data collection and address any questions. This approach will minimise participant contact with the research team and clinicians. While self-nominated participants may be subject to participation bias, high completion rates and compliance are likely.

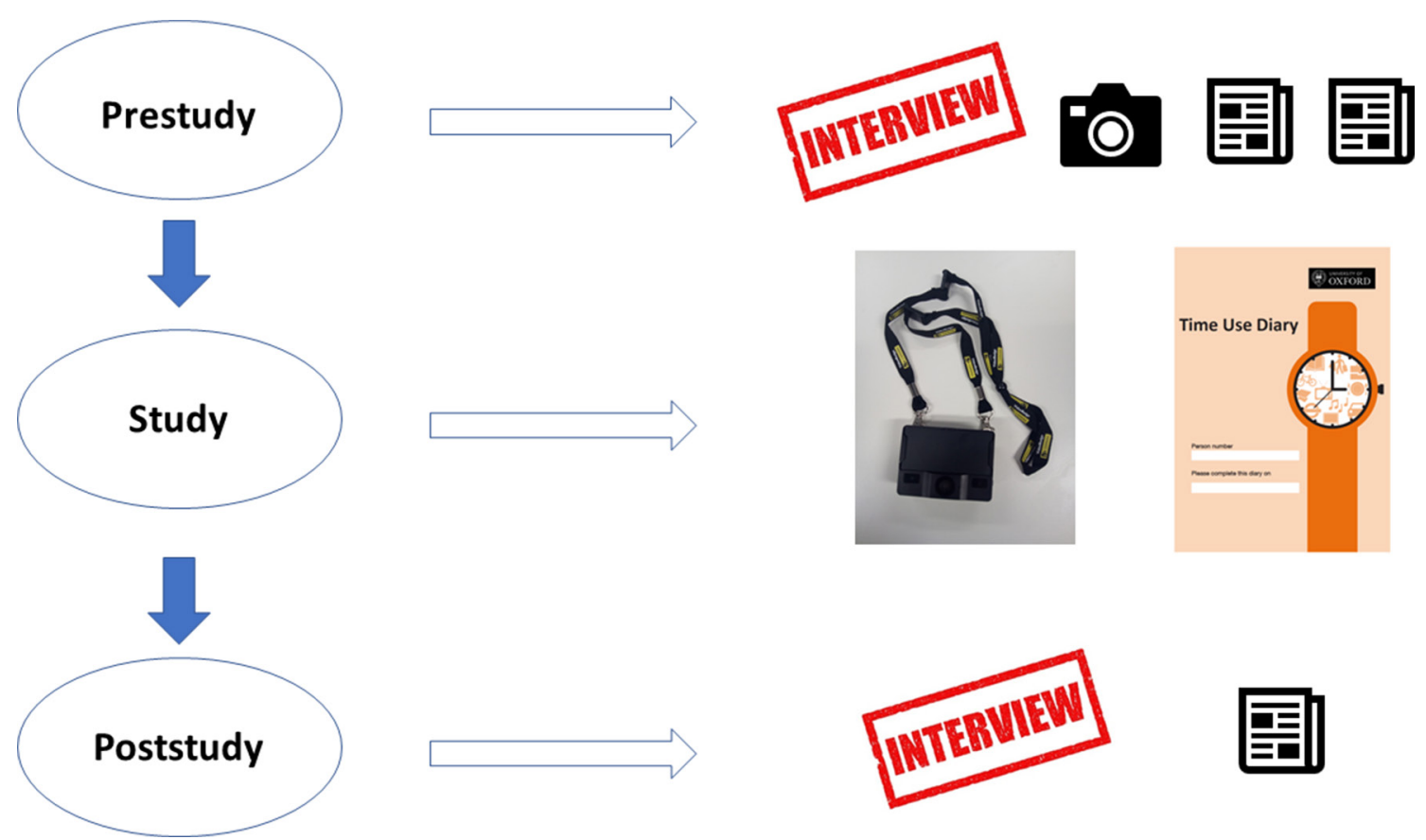

Figure 1 Flow diagram illustrating the tasks involved at each stage of the study. 
Table 1 Data collected at different stages during the study

\begin{tabular}{|c|c|c|}
\hline Prestudy & Data collection (24 hours) & Poststudy \\
\hline $\begin{array}{l}\text { Interview (1 hour) } \\
\text { Participant demographics questionnaire } \\
\text { Multimorbidity Illness Perceptions } \\
\text { Scale } \\
\text { - Photos of participant's home (where } \\
\text { appropriate) }\end{array}$ & $\begin{array}{l}\text { Body-worn camera } \\
\text { Time-use diary } \\
\text { (online supplementary appendix } \\
\text { 1) }\end{array}$ & $\begin{array}{l}\text { Reconstruction interview (30-40 min) } \\
\text { Participant experience Questionnaire } \\
\text { Researcher's notes }\end{array}$ \\
\hline
\end{tabular}

The following instruments and equipment will be used for data collection:

- Time-use diary. The self-report time-use diary is a validated too ${ }^{39}$ and the version used in this study is the Harmonised European Time-Use Diary ${ }^{42}$ (UK version). For details of the time-use diary, please see online supplementary appendix 2 . This method of using free-text time-use diaries to selfreport activities has high statistical validity and good acceptance rates with volunteer participants (ranging from $56 \%$ to $100 \%$ ). ${ }^{39} 46$

- Photos. With permission from the participant, photos of their medications, medical devices and home surroundings will be taken to inform about the participant's physical environment. Should the participants reside in an environment that can affect their healthcare (eg, a house with stairs and a risk for falls), these issues will be taken into consideration. Participants will be alerted should an immediate and significant risk be identified.

- Body-worn camera. The body-worn camera (Edesix VB-300, Edinburgh) will automatically record silent continuous video footage. It can be attached to clothing and/or worn on a lanyard. Previous studies with body-worn cameras showed high degrees of acceptability, with acceptance rates ranging from $70 \%$ to $100 \%,{ }^{39} 4647$ especially when participants are reassured they can remove the camera if they feel the recording may intrude on their privacy. ${ }^{39} 46$

- Demographic questionnaire. The demographic questionnaire (online supplementary appendix 3) will be administered during the prestudy session and takes about $10 \mathrm{~min}$ to complete.

- The Multimorbidity IIIness Perceptions Scale. The Multimorbidity Illness Perceptions Scale ${ }^{41}$ (online supplementary appendix 4) is validated statistically and was developed to capture the perception of patients with comorbidities about their health. ${ }^{41}$ IIIness perception is known to affect and predict health-related behaviour, to the extent that patients who perceive their diseases as more controllable are more likely to engage in adaptive strategies, ${ }^{48}$ thus directly affecting the patient work they engage in. The Scale will be administered during the prestudy session and takes approximately $5 \mathrm{~min}$ to complete.

- Participant experience questionnaire. A participant experience questionnaire (online supplementary appendix 5) will be administered during the poststudy session. This is estimated to take approximately $10 \mathrm{~min}$.

- Researcher notes. A summary of each participant will be written up within a week after the poststudy session, summarising the researcher's observations of the participant, home context and any feedback from the participant at different stages of the study.

\section{Patient and public involvement}

The concept study was conceived during a 1 day Consumer Symposium conducted at Macquarie University, where 10 health consumers gave feedback and critique regarding health research.

Health Consumers New South Wales, a patient advocacy group, was then consulted regarding consumer engagement and advice was given to the researchers regarding consumer involvement during study conception, recruitment, analysis to dissemination.

A pilot trial of the protocols with the equipment was conducted with two patients. Summaries of the findings were presented to the participants.

During study conception, the researchers also engaged with Healthdirect, a national organisation providing digital health information for health consumers, and the Northern Sydney Primary Health network and local GP network, which has a consumer council.

Results will be disseminated to these bodies, which will have broader implications to policies directed to consumers. Publications arising from the study will also be forwarded to each participant via emails.

\section{Data collection instruments and equipment}

The study will be carried out in three stages as described in figure 1 and table 1. Using multiple types of data, the study generates a holistic report of patient work conducted by people living with type 2 diabetes and chronic comorbidities in terms of observed physical work, organisational efforts, burden of disease and attitudes and beliefs underlying health behaviours.
The 24-hour data collection is more likely to capture a more representative day compared with previous patient work studies, which only captures patient activities for a few hours at most. While it will be hard to find a day that is truly representative of one's life, the researchers ask the participants to nominate a day for the study that the participants themselves consider to be 'normal'.

\section{Stage 1: prestudy session}

Prior to the prestudy session, participants will be provided with the Participant Information Booklet (online supplementary appendix 1) and will sign a written informed consent form (online supplementary appendix 6) before data are collected. Participants have the right to decline at this stage and to retract consent at any time throughout the study.

Ideally, the prestudy interview will occur in the participant's home for researchers to note ergonomic factors. To ensure researcher safety, two researchers will make the home visits. Participants may come to Macquarie University for the interview if they prefer.

Following consent and before the prestudy interview, participants will also be asked to complete the demographic questionnaire (online supplementary appendix 3) and the Multimorbidity Illness Perceptions Scale ${ }^{41}$ (online supplementary appendix 4).

\section{Prestudy interview}

After the completion of the questionnaires, a semistructured interview will be conducted to gain information about the participant's life history, medical history and 


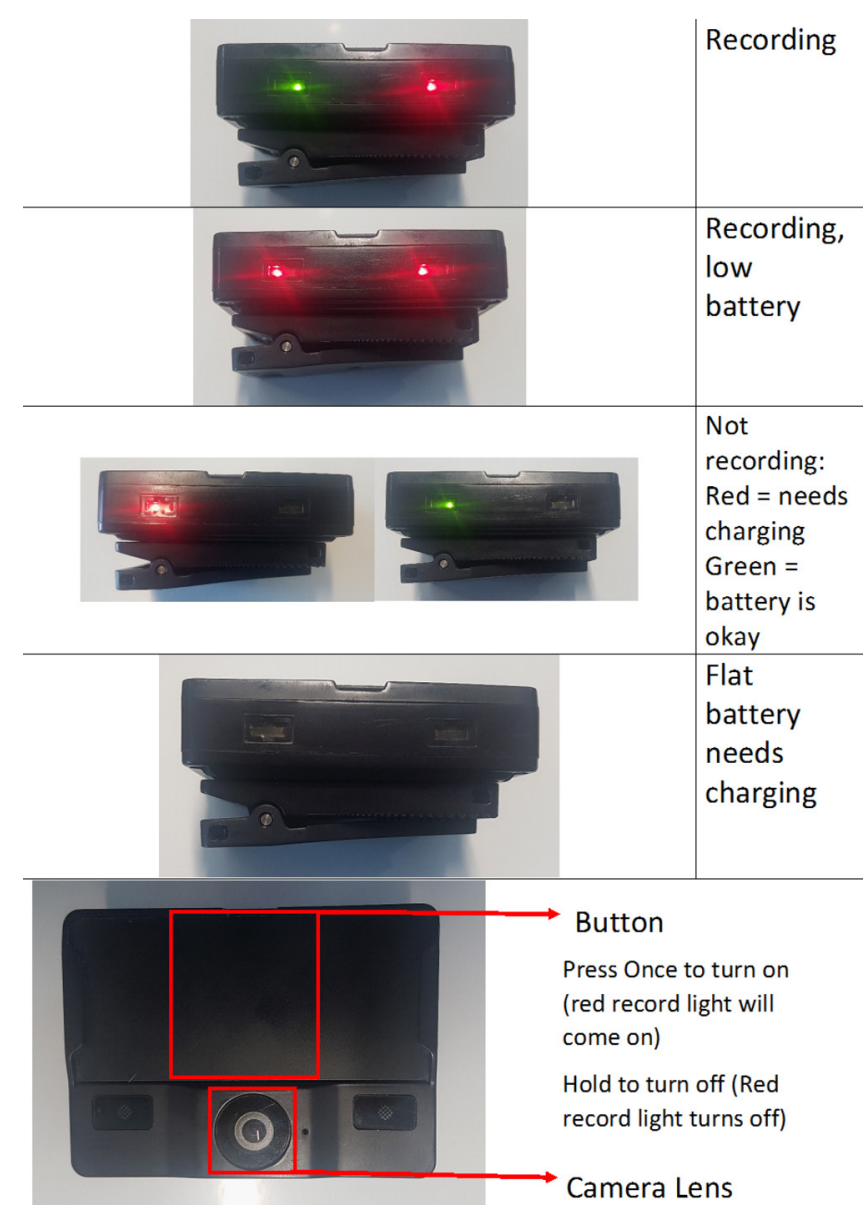

Figure 2 Instructions provided to participants regarding the operation of the body-worn camera.

existing factors that impact patient work. Participants will be invited to talk about their health and lifestyle, with areas of focus separated into life history, social life and mental health, physical health and medical history, physical environment, and organisational effort and attitude (online supplementary appendix 7). With the participant's permission, the interview will be audio recorded to assist with data coding and thematic analysis (eg, daily activities, rationales behind behaviours and factors that influence why participants behave in certain manners).

Lastly, participant will be provided with a body-worn camera and the Harmonised European Time Use Surveys UK Time-Use Diary (online supplementary appendix 2), ${ }^{42}$ together with relevant instructions. Photographs of the participant's medications, medical devices and home environment will also be taken at this stage.

\section{Stage 2: participant self-recording data collection}

During the 24-hour study period, participants will be asked to wear the camera around their neck on a lanyard during waking hours (12-16 hours). The time-use diary will be completed by the participant over the course of the same day for 24 hours. Participants will be given instructions (figure 2) to recharge the camera overnight or if the battery becomes low.

\section{Stage 3: poststudy session}

A poststudy session will occur immediately after the 24-hour study period has concluded. The camera footage will be downloaded and converted into images using software developed in-house, with one screenshot taken for every $10 \mathrm{~s}$ of footage. The participants will be able to view all the images alone before the research team views them, allowing the participant to delete any or all unwanted images.

\section{Poststudy 'reconstruction' interview}

The participant will be interviewed for approximately 30-40 min after data collection (online supplementary appendix 8). Participants will be asked to describe the study day, using the screenshots and time-use diary as prompts, with a focus on health-related tasks. The interview will be audio recorded with the participant's permission to allow for thematic analysis of transcripts.

During the second interview, the researchers will also ask the participant whether the study day was 'normal' for them, and whether anything special occurred.

After the interview, the participant will be asked to complete the participant experience questionnaire (online supplementary appendix 5) and provide feedback on the methodology.

\section{DATA ANALYSIS}

Both qualitative and quantitative data will be gathered and analysed. The varied data sources are complementary and focus on different aspects of patient work. For example, self-reported data regarding daily tasks via the time-use diary can be enriched through an examination of the screenshots from the camera.

\section{Quantitative data analysis}

Video recordings from the body-worn camera will be automatically converted into series of $10 \mathrm{~s}$ screenshots, which are then processed for analysis of daily activities (eg, eating, sleeping). These screenshots will be tagged using the TensorFlow ${ }^{43}$ and/or Clarifai (New York, USA) Application Programming Interface, which automatically generates tags that identify objects and describe content within the image (eg, a woman and a car). A subset of these tags will be manually verified for accuracy.

Time-use diary data will be analysed in conjunction with camera screenshots. Participants' activities reported in the time-use diary and observed via screenshots will be coded using an adapted version of the Harmonised European Time Use Surveys activity frame, ${ }^{44}$ and assessed for the duration, frequency, timing and patterns.

Questionnaire data (participant demographics, study feedback and the Multimorbidity Illness Perceptions Scale) will be reported using descriptive and inferential statistics. All statistical analyses will be conducted using SPSS v.25 (IBM).

\section{Qualitative data analysis}

Both prestudy and poststudy interviews will be transcribed verbatim. Analysis of the interview transcripts will 
be conducted using the NVivo thematic analysis software package (NVivo, Melbourne, Australia). ${ }^{45}$ Two members of the research team will independently code the transcripts in a systematic manner. Any coding discrepancies between the two will be resolved through reaching a consensus.

Photographs of the participant's medications, medical devices and home environment and camera screenshots will provide context to the interview. The photographs will also be used to give contextual feedback regarding the participant's daily activities, as revealed in the video recordings and time-use diaries. Notes taken by researchers after the poststudy interview for each participant will be used to help with photo and qualitative data analysis.

\section{ETHICS AND INFORMATION DISSEMINATION}

Ethical considerations arising from this study are addressed below.

\section{Consent and freedom to withdraw}

Participants' questions or concerns will be addressed individually and with all due diligence. Participants will be free to withdraw from the study at any point and can remove the camera at any time during the study day without giving a reason. The decision to join the study will have no consequence on their relationship with either Macquarie University or their health professionals.

\section{Third-party and bystander consent}

The research team will provide participants with a walletsized card that includes the statement below with contact information of the research team, which can be shown to others (to reduce the psychological burden of the participant) should anyone approach the participants to ask about the camera.

I am volunteering for a research project. The device is a wearable camera and the images will be used to record my daily activities. I am happy to remove it if you would like me to.

Should anyone request video recordings to be deleted, we ask participants to take note of the time that requests were made, and to assure the enquirer that the related recordings will be removed at the poststudy session. Participants are also asked to inform their household members, friends and acquaintances about the study, and to seek permission from these parties prior to recording.

\section{Personal privacy}

Participants will be asked to keep the body-worn camera off during situations when anyone (including themselves) is dressing, undressing, using the bathroom, showering or during any situations where privacy is of concern. Participants will be advised that they should always prioritise their own personal safety and comfort above the study's requirements.
Furthermore, participants will be able to view and delete any (or all) of the extracted images before researchers view the images or videos at the start of the poststudy session. The videos will be deleted immediately after screenshots are extracted. Only members of the research team will have access to the screenshots. None of the video or screenshots will be released to the public, academic peers or participants. Should participants provide written informed consent for the research team to use their images in publications or presentations, the images will be blurred such that no one is identifiable.

To ensure the participant's privacy, names and other identifying information will be removed in the photos of medications.

\section{Participant feedback}

Participants will be asked about their experiences in the study during the poststudy interview. A questionnaire to assess the participant's experience (online supplementary appendix 5) will also be administered. Participants will be provided with contact details of the research team (email and phone number) for contact during the study.

\section{Psychological support}

In the unlikely case that participants become psychologically distressed during either of the interview sessions, a staff member with counselling experience will be present in both interviews to deal with any anxiety the participant may experience. Should participants experience high levels of distress during the study day, they are advised that they should immediately attend their local hospital emergency department and GP, and/or call Lifeline or emergency services.

\section{Participant identification}

Participant identities will not be masked during data collection. Participants will be individually identifiable due to the small sample size. However, their identities will only be known to the research team, and only members of the research team will have access to identifiable data. Data will be separated from contact details and stored in separate locations. No identifying information about the participant will be published or presented.

\section{Information dissemination}

Great caution will be taken in the handling and storage of the data by the research team so that risks to privacy are minimised, in accordance with the Australian Code for the Responsible Conduct of Research. Only members of the research team will have access to the data. The videos will be downloaded into a secure browser in a password-protected computer or Macquarie University servers. All physical copies of surveys and questionnaires will be locked in secure cabinets at Macquarie University. The data will be held for a minimum of 5 years. Anonymity will be upheld throughout reporting, and participants will only be referred to by their participant number. 


\section{DISCUSSION}

At the time of writing, the study had engaged 40 potential participants and 23 participants completed the study, more than half of the number of participants the study aims for.

The reasons some potential participants did not take part in the study included: declined consent due to personal reasons (which the participant did not disclose), unable to communicate in English to a sufficient standard (as judged by the researcher during the initial phone call), too busy with work, too busy due to full-time care of family members, going on a holiday, was sick on the scheduled visit and withdrew from the study, and was not home when the researchers visited and withdrew from the study. No participant who completed the study had withdrawn their consent to date.

Completion rates for the time-use diary is very high, with 21 out of 23 participants successfully filling the diary throughout the study day. The two participants who did not fill in the diary did so during the poststudy interview, with the assistance of recorded footage.

All participants complied with continuous camera recording. There was a variation of camera recording time between participants, due to some participants turning the camera off for some periods of time out of privacy concerns.

One challenge the study had faced was communicating camera operations effectively to the participants. Some participants had difficulty turning off the camera and charging the camera battery due to reasons such as forgetfulness and confusion regarding the orientation of the camera buttons. To address the issue, the research team had devoted more time during the prestudy interview to physically demonstrate how to use the camera, which had successfully improved participant understanding of camera operations.

Acknowledgements Protocols: The authors of this paper acknowledge the researchers who designed and carried out the CAPTURE-24 Project carried out at the University of Oxford: Teresa Harms (University of Oxford, Department of Sociology, Centre for Time Use Research, Oxford, UK), Jonathan Gershuny (University of Oxford, Department of Sociology, Centre for Time Use Research, Oxford, UK), Aiden Doherty (Nuffield Department of Population Health, University of Oxford, UK), Emma Thomas (Melbourne School of Population and Global Health, University of Melbourne, Australia), Karen Milton (Norwich Medical School, University of East Anglia, Norwich, UK), Charlie Foster (School for Policy Studies, University of Bristol, UK). Technical: The screenshot extraction and tagging system was developed in-house by Mr Wen-Jun Liang and Mr Joshua Haddrill, two work placement students from the Faculty of Engineering at Macquarie University. Study: Mr Joshua Jung, Ms Isabelle Barnes, Mr Jiawei He and Mr Christian Tran (work placement students from Macquarie University) assisted with participant recruitment, home visits and administrative tasks. Participant recruitment: Professor Bernard Tuch and Dr Priscilla Wong, who assisted with recruitment at the early stages of the study. Drafting the manuscript: Dr Theun Pieter van Tienoven from the University of New South Wales, for his valuable comments and suggestions on earlier drafts of the manuscript. The authors thank the study participants for their valuable time and input.

Contributors Study conceptualisation: AYSL, EC. Study design: AYSL, KY, TH, JG. Data collection preparation and analysis plan: KY, TH, AYSL, LL, FR. Recruitment and liaison: KH, SV. First draft: KY, AYSL. Draft revision: All authors provided feedback and approved the manuscript.
Funding The project is supported by the New South Wales Health Early-to-Mid Career Research Fellowship awarded to AYSL.

Disclaimer The funding body did not have a role in study design and execution; data collection, management, analysis and interpretation; or manuscript preparation, review or approval.

Competing interests Some participants may be recruited via the Macquarie Health General Practitioner clinic and Macquarie Hospital, where SV is practising as a general practitioner and $\mathrm{KH}$ is practising as an endocrinologist, respectively. Therefore, the two team members may be responsible for the treatment of some participants. However, SV and KH will not know whether a patient is a participant unless the participant informs them.

Patient consent for publication Not required.

Ethics approval Macquarie University Human Research Ethics Committee (approval number 5201700718).

Provenance and peer review Not commissioned; externally peer reviewed.

Open access This is an open access article distributed in accordance with the Creative Commons Attribution Non Commercial (CC BY-NC 4.0) license, which permits others to distribute, remix, adapt, build upon this work non-commercially, and license their derivative works on different terms, provided the original work is properly cited, appropriate credit is given, any changes made indicated, and the use is non-commercial. See: http://creativecommons.org/licenses/by-nc/4.0/.

\section{REFERENCES}

1. Lorig KR, Holman H. Self-management education: history, definition, outcomes, and mechanisms. Ann Behav Med 2003;26:1-7.

2. Kaptein AA, Fischer MJ, Scharloo M. Self-management in patients with COPD: theoretical context, content, outcomes, and integration into clinical care. Int J Chron Obstruct Pulmon Dis 2014;9:907-17.

3. Apps LD, Harrison SL, Williams JE, et al. How do informal self-care strategies evolve among patients with chronic obstructive pulmonary disease managed in primary care? A qualitative study. Int $J$ Chron Obstruct Pulmon Dis 2014;9:257-63.

4. Bodenheimer T, et al. Patient self-management of chronic disease in primary care. JAMA 2002;288:2469-75.

5. Clark NM, Gong M, Kaciroti N. A model of self-regulation for control of chronic disease. Health Educ Behav 2001;28:769-82.

6. Liddy C, Blazkho V, Mill K. Challenges of self-management when living with multiple chronic conditions: systematic review of the qualitative literature. Can Fam Physician 2014;60:1123-33.

7. Koch G, Wakefield BJ, Wakefield DS. Barriers and facilitators to managing multiple chronic conditions: a systematic literature review. West J Nurs Res 2015;37:498-516.

8. Ong BN, Jinks C, Morden A. The hard work of self-management: living with chronic knee pain. Int J Qual Stud Health Well-being $2011 ; 6$.

9. Yen L, McRae IS, Jowsey T, et al. Health work by older people with chronic illness: how much time does it take? Chronic IIIn 2013;9:268-82.

10. van Houtum L, Rijken M, Groenewegen P. Do everyday problems of people with chronic illness interfere with their disease management? BMC Public Health 2015;15:1000.

11. Cheraghi-Sohi S, Morden A, Bower P, et al. Exploring patient priorities among long-term conditions in multimorbidity: A qualitative secondary analysis. SAGE Open Med 2013;1:1.

12. Dwarswaard J, Bakker EJ, van Staa A, et al. Self-management support from the perspective of patients with a chronic condition: a thematic synthesis of qualitative studies. Health Expect 2016;19:194-208.

13. Sadler E, Wolfe CD, McKevitt C. Lay and health care professional understandings of self-management: a systematic review and narrative synthesis. SAGE Open Med 2014;2:205031211454449.

14. Thille P, Ward N, Russell G. Self-management support in primary care: enactments, disruptions, and conversational consequences. Soc Sci Med 2014;108:97-105.

15. Crowley MJ, Holleman R, Klamerus ML, et al. Factors associated with persistent poorly controlled diabetes mellitus: clues to improving management in patients with resistant poor control. Chronic IIIn 2014;10:291-302.

16. Free C, Phillips G, Galli L, et al. The effectiveness of mobile-health technology-based health behaviour change or disease management interventions for health care consumers: a systematic review. PLoS Med 2013;10:e1001362. 
17. Alzubaidi H, Mc Namara K, Browning C. Time to question diabetes self-management support for Arabic-speaking migrants: exploring a new model of care. Diabet Med 2017;34:348-55.

18. Schinckus L, Dangoisse F, Van den Broucke S, et al. When knowing is not enough: emotional distress and depression reduce the positive effects of health literacy on diabetes self-management. Patient Educ Couns 2018;101:324-30.

19. Valdez RS, Gibbons MC, Siegel ER, et al. Designing consumer health IT to enhance usability among different racial and ethnic groups within the United States. Health Technol 2012;2:225-33.

20. Farvolden P, Denisoff E, Selby P, et al. Usage and longitudinal effectiveness of a Web-based self-help cognitive behavioral therapy program for panic disorder. J Med Internet Res 2005;7:e7

21. Eysenbach G. The law of attrition. J Med Internet Res 2005;7:e11.

22. Yu CH, Bahniwal R, Laupacis A, et al. Systematic review and evaluation of web-accessible tools for management of diabetes and related cardiovascular risk factors by patients and healthcare providers. J Am Med Inform Assoc 2012;19:514e22.

23. Valdez RS, Holden RJ, Novak LL, et al. Transforming consumer health informatics through a patient work framework: connecting patients to context. J Am Med Inform Assoc 2015;22:2-10.

24. Mickelson RS, Holden RJ. Medication management strategies used by older adults with heart failure: a systems-based analysis. Eur $J$ Cardiovasc Nurs 2018;17.

25. Mickelson RS, Unertl KM, Holden RJ. Medication Management: The Macrocognitive Workflow of Older Adults With Heart Failure. JMIR Hum Factors 2016;3:e27.

26. Khunti K, Davies MJ, Kalra S. Self-titration of insulin in the management of people with type 2 diabetes: a practical solution to improve management in primary care. Diabetes Obes Metab 2013;15:690-700.

27. Holden RJ, Valdez RS, Schubert CC, et al. Macroergonomic factors in the patient work system: examining the context of patients with chronic illness. Ergonomics 2017;60:26-43.

28. Holden RJ, Schubert CC, Mickelson RS. The patient work system: an analysis of self-care performance barriers among elderly heart failure patients and their informal caregivers. Appl Ergon 2015;47:133-50.

29. Carayon P, Karsh BT, Gurses AP, et al. Macroergonomics in Healthcare Quality and Patient Safety. Rev Hum Factors Ergon 2013;8:4-54.

30. Heiden SM, Holden RJ, Alder CA, et al. Human factors in mental healthcare: a work system analysis of a community-based program for older adults with depression and dementia. Appl Ergon 2017:64:27-40

31. McCambridge J, Witton J, Elbourne DR. Systematic review of the Hawthorne effect: new concepts are needed to study research participation effects. J Clin Epidemiol 2014;67:267-77.
32. Khazrai YM, Defeudis G, Pozzilli P. Effect of diet on type 2 diabetes mellitus: a review. Diabetes Metab Res Rev 2014;30:24-33.

33. Wilding JP. The importance of weight management in type 2 diabetes mellitus. Int J Clin Pract 2014:68:682-91.

34. Balducci S, Sacchetti M, Haxhi J, et al. Physical exercise as therapy for type 2 diabetes mellitus. Diabetes Metab Res Rev 2014;30:13-23.

35. Costa LA, Canani LH, Lisbôa HR, et al. Aggregation of features of the metabolic syndrome is associated with increased prevalence of chronic complications in Type 2 diabetes. Diabet Med 2004;21:252-5

36. Bosi E, Scavini M, Ceriello A, et al. Intensive structured selfmonitoring of blood glucose and glycemic control in noninsulintreated type 2 diabetes: the PRISMA randomized trial. Diabetes Care 2013;36:2887-94.

37. Wasem J, Bramlage P, Gitt AK, et al. Co-morbidity but not dysglycaemia reduces quality of life in patients with type-2 diabetes treated with oral mono- or dual combination therapy--an analysis of the DiaRegis registry. Cardiovasc Diabetol 2013;12:47.

38. Kannan H, Thompson S, Bolge SC. Economic and humanistic outcomes associated with comorbid type-2 diabetes, high cholesterol, and hypertension among individuals who are overweight or obese. J Occup Environ Med 2008;50:542-9.

39. Kelly P, Thomas E, Doherty A, et al. Developing a method to test the validity of 24 hour time use diaries using wearable cameras: a feasibility pilot. PLoS One 2015;10:e0142198.

40. Kelly P, Marshall SJ, Badland $\mathrm{H}$, et al. An ethical framework for automated, wearable cameras in health behavior research. $A m \mathrm{~J}$ Prev Med 2013;44:314-9.

41. Gibbons CJ, Kenning C, Coventry PA, et al. Development of a multimorbidity illness perceptions scale (MULTIPIeS). PLoS One 2013;8:e81852.

42. Eurostat. Harmonised European time use surveys 2008 guidelines. Luxembourg: Eurostat, 2009.

43. Abadi M, Agarwal A, Barham P, et al. Tensorflow: large-scale machine learning on heterogeneous distributed systems. 2016.

44. Oxford Uo. Multinational time use study - user's guide and documentation. 2012.

45. Braun V, Clarke V. Using thematic analysis in psychology. Qual Res Psychol 2006;3:77-101.

46. Kelly P, Doherty A, Mizdrak A, et al. High group level validity but high random error of a self-report travel diary, as assessed by wearable cameras. J Transp Health 2014:1:190-201.

47. Doherty AR, Kelly P, Kerr J, et al. Using wearable cameras to categorise type and context of accelerometer-identified episodes of physical activity. Int J Behav Nutr Phys Act 2013;10:22.

48. Hagger MS, Koch S, Chatzisarantis NLD, et al. The common sense model of self-regulation: Meta-analysis and test of a process model. Psychol Bull 2017;143:1117-54. 